\title{
Use of a multidisciplinary tool to achieve target outcomes in Native American patients with diabetes: Treat-to-target
}

\author{
Emily L Sexson \\ Michael S Monaghan \\ Thomas L Lenz \\ Ann Ryan Haddad \\ Gail Jensen \\ Gary Elsasser \\ Creighton University School \\ of Pharmacy and Health Professions, \\ Omaha, NE, USA
}

\begin{abstract}
Purpose: Our purpose was to test a communication tool used in a multidisciplinary setting to more effectively achieve the recommended goals for glucose, blood pressure, lipids, and prophylactic aspirin use in a Native American population with type 2 diabetes.

Methods: One hundred randomly selected patients were included in this observational, preintervention, post-intervention study design. The team began with a chart audit documenting hemoglobin $\mathrm{A}_{1 \mathrm{c}}\left(\mathrm{Hgb} \mathrm{A}_{1 \mathrm{c}}\right)$, blood pressure, cholesterol levels, and aspirin use. The intervention included the development of a one page form used to prompt providers to intensify therapy when the patient was not meeting evidence-based goals. The audit was repeated one year later.

Results: Analysis of 74 patients completing the study showed a decrease in Hgb A1C from $8.812 \%$ pre-intervention to a mean $8.214 \%$ post-intervention $(\mathrm{p}<0.007)$. At the time of preintervention audit, patients were already at target for blood pressure and no significant further decrease was found. Measures of total cholesterol, triglycerides, and aspirin use showed improvement, but statistical significance was not met.

Conclusion: The one-page multidisciplinary tool used to intensify therapy significantly improved glucose control. More consistent interaction of the multidisciplinary team is necessary to reach other desired goals.
\end{abstract}

Keywords: diabetes, multidisciplinary, treat-to-target, Native American

\section{Introduction}

In recent years, the overall incidence of cardiovascular disease (CVD) has been on the rise among Native American populations (Howard et al 1999). It is likely that there are not only genetic differences resulting in varied responses to therapy in Native Americans, but also an issue of decreased access to optimal care. A closer examination by the Centers for Disease Control and Prevention reveals that $61 \%-64 \%$ of Native Americans currently have one or more risk factors that lead to the development of cardiovascular disease (CVD) (CDC 2000).

Diabetes has reached epidemic proportions among Native Americans. On average, Native Americans are 2.2 times more likely to be diagnosed with diabetes than are nonHispanic white cohorts of the same age. In 2003, nearly $13 \%$ of the 99,500 American Indians and Alaska Natives aged 20 years or older who received care from the Indian Health Service (IHS) in 2003 had been diagnosed with diabetes (IHS 2007). Increases in cardiovascular risk factors for Native Americans may be due to changes in lifestyle (decreases in activity level and exercise) and consumption of a more westernized diet with foods high in salt, fat, and cholesterol (Gilbert et al 1992). In addition, while complications such as retinopathy, neuropathy, and nephropathy related to diabetes exist and may worsen patient outcomes, the cardiovascular complications of diabetes (coronary heart disease, stroke, and congestive heart failure) consistently increase the 
risk of death from the disease. The IHS report that Native Americans with diabetes are two to three times more likely to develop heart disease than those without diabetes. It has been reported that nearly $80 \%$ of the mortality associated with diabetes has a cardiovascular morphology (Frey et al 1998; NDIC 2007).

Considering the prevalence of diabetes and the negative outcomes that result from the disease, a patient's entire health care team should consider prevention strategies to slow the incidence of diagnoses in all populations. When considering risk factors for developing CVD, hypertension and hyperlipidemia are less prevalent among Native Americans. Their existence, however, when added to existing hyperglycemia, continue to be major risk factors for the development of CVD (Frey et al 1998; Harwell et al 2001; Burden et al 2002).

As CVD continues to be a leading cause of death among Native Americans with diabetes, it is imperative that health care practitioners assess these major risk factors and develop systematic approaches for improving treatment and prevention efforts (Howard 1996; CDC 2000; Harwell et al 2001). Evidence-based practice suggests that control over major CVD risk factors could be key to reducing morbidity and mortality associated with diabetes. In patients with limited access such as the Native American population studied, each health care provider who comes in contact with the patient should utilize a communication tool to relay important information about the patient's health status to all essential members of the health care team in order to provide optimal care.

Our purpose in designing this study was to develop a tool that could be used by members of the multidisciplinary team to more effectively institute guideline-based medicine that optimizes the patient's pharmacotherapy and achieves their health outcome goals.

\section{Methods}

The one-page tool we developed for use was named Treat-to-Target (see Appendix 1). The Treat-to-Target approach aimed at intensifying pharmacologic therapy for the purpose of improving the patients' overall control of CVD risk factors.

The patients enrolled in Treat-to-Target were being seen at a local outpatient clinic that provides services primarily to the community members residing on the reservation. Once a strong need for improvement of patient care was identified by health care providers from Creighton University and those employed on the reservation, our plan was implemented.
This study used an observational, pre-intervention, post-intervention study design. One hundred Native Americans with type 2 diabetes were randomly selected from a diabetes center patient population. A pre-intervention chart review was performed which included documentation of the following: current hemoglobin $\mathrm{A}_{1 \mathrm{c}} \mathrm{c}\left(\mathrm{Hgb} \mathrm{\textrm {A } _ { 1 \mathrm { c } }}\right)$ value, blood pressure, cholesterol levels, and use of preventive or prophylactic aspirin. The review also recorded current medications and used the one-page Treat-to-Target form to recommend opportunities for improvement in disease state of which the patient was not meeting evidence-based goals. For example, if a chart indicated that a patient currently had an $\mathrm{Hgb} \mathrm{A}_{1 \mathrm{c}}$ value of $8.7 \%$ and was taking metformin $1 \mathrm{~g}$ twice daily and glipizide $10 \mathrm{mg}$ twice daily, step three on the Treat-to-Target tool would be circled, recommending the addition of pioglitazone (see Appendix 1). The Treat-to-Target sheet was placed in the patient's medical chart for the prescriber to see at the next patient visit.

Of note, the Treat-to-Target intervention used a lowdensity lipoprotein (LDL) goal of $<100$ to ensure at least the recommended goal for patients with diabetes was met. No recommendations were made for further reduction after that goal was met.

Immediately following the first audit, the intervention was performed. It consisted of an educational inservice to the medical and nursing staff at the diabetes clinic. The inservice explained the development of the Treat-to-Target approach to intensify drug therapy to reach specific goals and cited the source of the recommendations as those of the American Diabetes Association (ADA) (2008). The presentation to the medical staff delineated the outcomes evidence behind these guidelines that led to the target goals, and explained how this sheet could be used in the current clinic structure to aid in achieving target goals for glucose, blood pressure, lipids, and aspirin use.

Approximately 13 months later, a post-intervention chart review was repeated in which the patients served as their own controls. The same data were collected as before: current $\mathrm{Hgb}$ A1C value, blood pressure, LDL cholesterol value, and use of aspirin. A paired t-test comparison was made for each pre-post data set for the $\mathrm{Hgb} \mathrm{A}_{1 \mathrm{c}}$ values, LDL cholesterol values, and aspirin use data, in which $\mathrm{p}<0.05$ was considered significant.

\section{Results}

A total of 73 participants had complete data. Due to nonnormal distributions, nonparametric statistics were used. For continuous data, the Wilcoxon signed-ranks test was 
used, while the McNemar test was used for nominal data. A Bonferroni adjustment adjusted $\alpha=0.007$ to reduce the probability of type 1 error from multiple analyses of the same data.

Data about each collected parameter is summarized in Table 1 . Hgb $A_{1 c}$ values decreased from a mean $8.812 \%$ pre-intervention to a mean $8.214 \%$ post-intervention $(\mathrm{p}<0.007)$. Patients were meeting the ADA's goals for systolic and diastolic blood pressure before the Treat-to-Target tool was initiated with a pre-intervention mean of $128.1 / 72.68 \mathrm{~mm} \mathrm{Hg}$. Slight reductions in both systolic and diastolic levels were found post-intervention (though not statistically significant) with mean blood pressure being 127.11/71.11 ( $\mathrm{p}=0.47)$. Neither total cholesterol nor triglyceride levels decreased significantly, though reductions were noted in both. Insufficient LDL cholesterol values were drawn for analysis. Using the McNemar test to evaluate aspirin use pre-and post-intervention, nonsignificant increases in the number of patients on aspirin were found $(p=0.503)$.

\section{Discussion}

Current practice in the treatment of diabetes and cardiovascular disease does not offer a standard tool for adjusting pharmacotherapy. There are many considerations that go into making alterations in patient medications and our study has provided a tool that could provide any health care provider the ability to make consistent evidence-based decisions each time the patient was seen. We believe this would likely lead to improved patient outcomes.

The environment this tool was piloted in was a rural, Native American outpatient clinic in which a multidisciplinary approach to treating patients was used frequently. Interventions made by all members of the healthcare team were expected to provide the patient with optimal care. The ability to make recommendations directly to the provider through a previous chart review was appreciated by the providers as they were able to have maximal impact on patients they saw in clinic despite short appointment times.

Using a chart review process and incorporation of the Treat-to-Target approach by a multidisciplinary team was a successful method to reduce $\mathrm{Hgb} \mathrm{A}_{1 \mathrm{c}}$ values and helped to align patients closer to other goals outlined in the ADA standards for treating diabetes. The results, however, indicate that a more intensive effort is needed in the areas of achieving cholesterol and aspirin use goals.

Cholesterol monitoring and assessment of adherence to daily aspirin was not performed at frequent intervals to ensure goals were met. More frequent monitoring may have been accomplished through improved incorporation of other members of a multidisciplinary team that may have more access to the patient. Our study used pharmacists only to review the patient's chart and make recommendations. However, if each provider active in caring for the patient was prompted to utilize the algorithm developed each time the patient was seen, improved compliance to medications, laboratory draws, and more frequent evaluations of those results may have resulted in more positive outcomes.

As patients are multifaceted individuals, so are their disease states. There are many modifications necessary in addition to drug therapy that will affect their ability to obtain goals. Exploration into the role that health care professionals on the multidisciplinary team could play in these efforts would also be beneficial in developing a more holistic approach to managing the disease. An example of this could include discussion of lifestyle modifications recommended by the ADA. As westernization of Native American diets has been cited as a contributing factor to the development of diabetes, ensuring that patients meet with providers trained in dietary counseling could be essential to improving outcomes. Non-pharmacologic methods of reducing risk of developing CVD were not included on the Treat-to-Target tool, and data comparing differences in patients' knowledge and use of those methods was not collected.

Table I Results from Treat-To-Target

\begin{tabular}{llllll}
\hline & N Pre-intervention & Pre-intervention mean & N Post-intervention & Post-intervention mean & P value \\
\hline Hgb A & 73 & $8.812 \%$ & 70 & $8.214 \%$ & $0.007^{*}$ \\
Systolic blood pressure & 72 & 128 & 72 & 127 & 0.47 \\
Diastolic blood pressure & 72 & 72 & 72 & 71 & 0.096 \\
Total cholesterol & 72 & 199 & 67 & 192 & 0.179 \\
Triglycerides & $7 \mathrm{I}$ & 266 & 66 & 255 & 0.236 \\
\hline
\end{tabular}

Abbreviation: Hemoglobin, $A_{\mid c} \mathrm{Hgb} A_{1 c}$. Note: *statistical significance. 
Another limitation that may have affected the results of our study is the lack of knowledge of the patients' medication history unrelated to those treating their diabetes, hypertension, hypercholesterolemia, or aspirin. It is not known if patients were on other antiplatelet or anticoagulant agents that may have limited aspirin use in an attempt to lower risk of bleeding. It is also not known if patients may have been taking a medication that may have increased their risk for developing metabolic syndrome (atypical antipsychotics).

Future efforts must also address guidelines regarding recommended annual measurements necessary to prevent or monitor diabetes related complications such as nephropathy, neuropathy, retinopathy, and others. Cardiovascular disease was the only outcome the study aimed at preventing. Patients with diabetes, however, should have all aspects of their health considered when seen by their health care professionals and incorporating these guidelines into the tool would be beneficial in preventing those negative outcomes from occurring.

Differences in the patient population could have affected compliance with taking the medications recommended. Baseline demographics were not gathered in this study. Cultural competency training for all providers may impact patient interest and compliance with the regimens and should be recommended in this population. This could be extrapolated to other settings with the diversity that exists in our communities today.

Despite these limitations, the significant improvement in glycemia seen with our one-page communication tool used to review the patient's medical chart validates the importance of working with a multidisciplinary team to achieve the common goal of optimal patient care. As only pharmacists and primary care providers used the Treat-to-Target tool in our study, we have also demonstrated the need to involve the entire team, including all health care professionals the patients see, to achieve an even greater impact on reducing progression to CVD. We believe our study demonstrates the fact that if it became the responsibility of not only the primary care provider, but the mission of the entire patient care team in this setting, the epidemic of diabetes could be slowed.

\section{Disclosure}

The authors report no conflicts of interest in this work.

\section{References}

[ADA] American Diabetes Association. 2008. Standards of medical care in diabetes 2008 [online]. Diabetes Care, 31:S12-S54. Accessed January 1, 2008. URL: http://care.diabetesjournals.org/content/vol31/ Supplement_1\%.

Burden RW, Kumar RN, Phillips DL, et al. 2002. Hyperlipidemia in Native Americans: evaluation of lipid management through a cardiovascular risk reduction program. $J$ Am Pharm Assoc, 42:652-5.

[CDC] Centers for Disease Control and Prevention. 2000. Prevalence of selected cardiovascular disease risk factors among American Indians and Alaska Natives. Morb Mortal Weekly Rep, 49:461-5.

Frey JL, Jahnke HK, Bulfinch EW. 1998. Differences in stroke between white, Hispanic and Native American patients: the barrow neurological institute stroke database. Stroke, 29:29-33.

Gilbert TJ, Percy CA, Sugarman JR et al. 1992. Obesity among Navajo adolescents: relationship to dietary intake and blood pressure. $\mathrm{Am} \mathrm{J}$ Dis Child, 146:289-95.

Harwell TS, Ghodes D, Moore K, et al. 2001. Cardiovascular disease risk factors in Montana American Indians and non-Indians. Am J Prev Med, 20:196-201.

Howard BV, Lee ET, Yeh JL, et al. 1996. Hypertension in adult American Indians: the strong heart study. Hypertension, 28:256-64.

Howard BV, Lee ET, Cowan LD, et al. 1999. The rising tide of cardiovascular in American Indians: the Strong Heart Study. Circulation, 99:2389-95.

[HIS] Indian Health Service; US Department of Health and Human Services. 2007. IHS best practice models; Cardiovascular disease and diabetes [online]. Accessed September 9, 2007. URL: http://www.ihs. gov/MedicalPrograms/Diabetes/Resources/cardioBP.pdf.

[NDIC] National Diabetes Information Clearinghouse. 2007. National diabetes statistics, 2007 fact sheet [online]. Accessed June 19, 2007. URL: http://diabetes.niddk.nih.gov/dm/pubs/statistics/index.htm. 


\section{Appendix I}

\section{Treat-to-Target Diabetes Review}

Circle recommended action

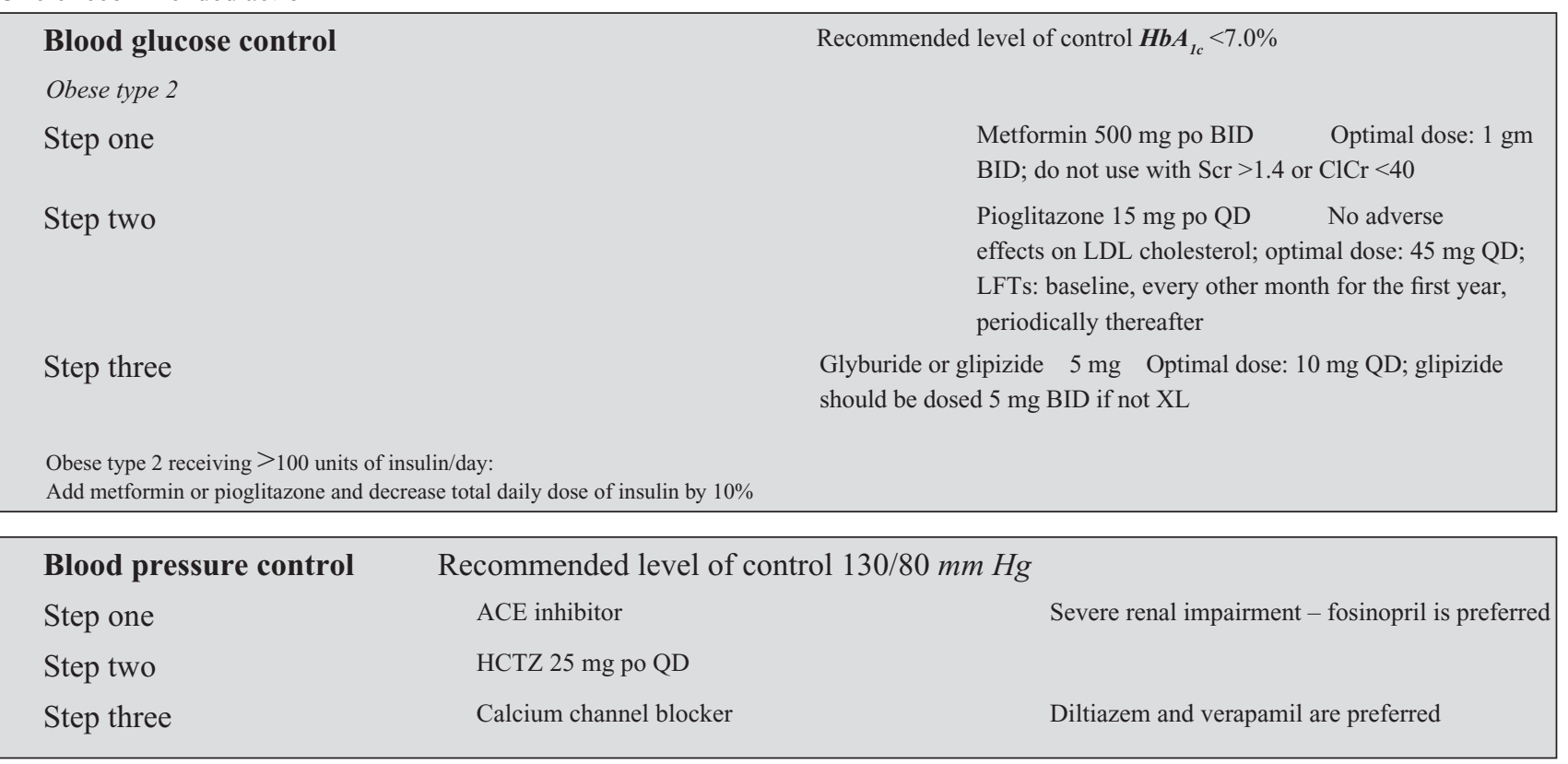

\section{Lipid control \\ $L D L$}

Step one

Step two

Triglycerides

Step one
Recommended level of control $<100 \mathrm{mg} / \mathbf{d L}$

Statin

Statin + fenofibrate Micronized fenofibrate $200 \mathrm{mg}$ QD with main meal; LFTs: baseline, every 3 months for the first year; if ALT $>100 \mathrm{IU} / \mathrm{L}$, DC therapy

Fenofibrate: $67 \mathrm{mg}$ QD with meal; max $67 \mathrm{mg}$ TID with meals

Add enteric-coated aspirin ( $75-162 \mathrm{mg} /$ day) for anyone $>30$ years with

A family history of heart disease

Hypertension

Albuminuria (micro or macro)

Lipids:

Cholesterol $>200 \mathrm{mg} / \mathrm{dL}$

Triglycerides $>200 \mathrm{mg} / \mathrm{dL}$

$\mathrm{HDL}<45 \mathrm{mg} / \mathrm{dL}$ (men) and $<55 \mathrm{mg} / \mathrm{dL}$ (women)

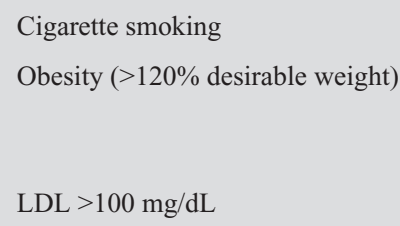

$\mathrm{d}<150 \mathrm{mg} / \mathrm{dL}$ Gemfibrozil or fenofibrate

. 
